\title{
Pratique d'Interdits Alimentaires: Entre Logique Identitaire, Enjeux Sanitaires Et Conservation De La Biodiversité Chez Les Agni De Bongouanou (Côte d'Ivoire)
}

\section{Koné Bognan Valentin, Doctorant}

Unité de Formation et de Recherche des Sciences de l'homme et de la Société, Université Félix Houphouët-Boigny, Abidjan, Côte d'Ivoire. Centre Suisse de Recherches Scientifiques en Côte d'Ivoire, Abidjan, Côte d'Ivoire, Université de Bâle, Suisse.

\section{Fokou Gilbert, Chercheur}

Centre Suisse de Recherches Scientifiques en Côte d'Ivoire, Abidjan, Côte d'Ivoire, Human Science Research Council, Cape Town, Afrique du Sud.

\section{Kouadio Baya Bouaki, Enseignant Chercheur}

Unité de Formation et de Recherche des Sciences de l'homme et de la Société, Université Félix Houphouët-Boigny, Abidjan, Côte d'Ivoire.

Brigit Obrist, Enseignant Chercheur

Institut d'Anthropologie Sociale, Université de Bâle, Suisse.

\section{Roch Yao Gnabeli, Enseignant Chercheur}

Unité de Formation et de Recherche des Sciences de l'homme et de la Société, Université Félix Houphouët-Boigny, Abidjan, Côte d'Ivoire.

\section{Bassirou Bonfoh, Chercheur}

Centre Suisse de Recherches Scientifiques en Côte d'Ivoire, Abidjan, Côte d'Ivoire.

\section{Doi: 10.19044/esj.2018.v14n27p82 URL:http://dx.doi.org/10.19044/esj.2018.v14n27p82}

\begin{abstract}
Social norms are depriving Agni communities of Bongouanou in central-eastern Côte d'Ivoire of certain foods adding to a state of food insecurity. Based on significant cases of food restrictions, this study analyzed nutritional practices that position individuals and groups in social normative frameworks. This work focused on institutional frameworks where health and food taboo aspects were most shared. This study aims to analyze the social imaginaries associated with food restriction and their link to health in a context of food insecurity. More particularly, it aims at exploring beliefs and representations associated with health risks due to food restrictions. Based on a qualitative approach using data collection techniques as direct observation,
\end{abstract}


semi-structured interviews and focus group discussions, this study was conducted in four villages where the consumption of catfish by local populations is totally prohibited. Social identity theory and the cultural materialism perspective were used for analysis and interpretation of knowledge and beliefs associated with food restrictions. The non-consumption of catfish from the Socotè Lake participated to sociability and the preservation of the indigenous "Agni of Bongouanou" identity. Additionally, food restrictions are determinants of physical and reproductive health of members of the social group. In fact, the collective imaginary of Agni from Bongouanou establishes a causal link between the consumption of this restricted food and health problems. Finally, it has emerged that this food practice could impact the cultural well-being of the community while contributing to the conservation of biodiversity.

Keywords: Food restriction, health, Agni community, Identity, Conservation, Bongouanou.

\section{Résumé}

Dans le contexte actuel d'insécurité alimentaire, des normes sociales privant les populations de certains aliments sont observées chez les Agni, groupe ethnique autochtone du département de Bongouanou au Centre-Est de la Côte d'Ivoire. En s'appuyant sur des cas significatifs d'interdits alimentaires, la présente étude analyse des pratiques nutritionnelles, qui inscrivent les individus et les groupes dans des cadres sociaux normatifs à contre-courant des logiques institutionnelles et socio-écologiques. Cette étude met en évidence les imaginaires sociaux associés à l'interdiction de consommer le poisson silure du lac Socotè de Bongouanou et leur rattachement aux problèmes de santé. L'étude a ciblé les populations de quatre villages et s'est appuyée sur des techniques de collecte des données qualitatives à savoir l'observation directe, les entretiens semi-structurés et des discussions de groupe. L'interprétation des données a été faite sur la base de la théorie de l'identité sociale et de la perspective du matérialisme culturel. Les résultats ont révélé que, ne pas consommer le poisson silure du lac Socotè, participe à la sociabilité, mais surtout à la construction et de la préservation de l'identité autochtone "Agni de Bongouanou". Ensuite, ces groupes sociaux ont construit un ensemble de règles comportementales attribuées à l'interdit alimentaire. Par ailleurs, l'imaginaire collectif de cette communauté établit un lien causal entre les problèmes sanitaires et la santé de reproduction avec le bris de ces interdits alimentaires. Enfin, la pratique d'interdit alimentaire contribue à la conservation de la biodiversité avec pour repercussion le bienêtre culturel des communautés. 
Mots clés: Interdits alimentaires, identité, santé, conservation, Agni de Bongouanou.

\section{Introduction}

Les effets de la pression environnementale sur la sécurité alimentaire mondiale ont été au centre des discussions à la conférence des Nations Unies sur les changements climatiques (COP21) de Paris en 2015. Aussi, le rapport de la FAO (2016), indique-t-il que des phénomènes tels que l'érosion, la sécheresse, les innondations, etc., pourraient compromettre les efforts au plan agricole pour nourrir les populations les plus vulnérables. Ce fait constituerait un frein aux progrès réalisés pour l'élimination de la faim et de la malnutrition. Face à cette situation, certains pays ont multiplié des stratégies et politiques pour apporter des réponses adéquates dans un contexte où l'accroissement de la population est inversement proportionnel à la production agricole.

A l'instar des autres pays Africains, la Côte d'Ivoire a pris plusieurs initiatives pour assurer la sécurité alimentaire et la sécurité sanitaire des aliments. Il s'agit entre autres de la création du Programme National de Nutrition (PNN) en 2001, du Conseil National pour la Nutrition (CNN) en 2014 et du Plan Multisectoriel de Nutrition 2016-2020 ${ }^{4}$. En plus, le Programme National d'Investissement Agricole (PNIA) a été initié en 2012 et dont la deuxième phase s'étend de 2017 à 2020. Des politiques sectorielles structurantes dont la Stratégie Nationale de Protection Sociale 2013-2015 ainsi que la Politique Nationale de l'Alimentation Scolaire 2012-2017 ont été définies. L'objectif à travers toutes ces actions, était d'améliorer l'état nutritionnel et sanitaire des populations issues des couches les plus vulnérables ${ }^{5}$. En outre, l'Etat Ivoirien a arrêté des mesures d'interdiction de consommation et d'importation de certaines protéines animales pour faire face à la survenue de la fièvre hémorragique à virus Ebola ${ }^{6}$, du virus du lac tilapia ${ }^{7}$, de l'émergence de la peste porcine ${ }^{8}$ au nord du pays et aux cas de grippes aviaires en Côte d'Ivoire (Yacouba, 2007). Ces mesures ont contribué à positionner la consommation du poisson et du champignon comestible comme

${ }^{4}$ http://www.nutrition.gouv.ci/conseil.php?lang=\&ID=3, consulté le 27 Novembre 2017.

${ }^{5}$ Les enfants, femmes enceintes, personnes du troisième âge, et les ménages à faible revenu.

${ }^{6}$ Le 21 mars 2014, la maladie découverte a été identifiée comme étant la maladie à virus Ebola. http://www.who.int/csr/disease/ebola/one-year-report/virus-origin/fr/

7 Arrêté $\mathrm{N}^{\circ} 033 / \mathrm{MIRAH} / \mathrm{CAB}$ du 08 Septembre 2017 portant interdiction provisoire d'importation, de commercialisation et/ou d'élevage de toute espèce de tilapia en provenance de la Colombie, de l'Equateur, l'Egypte, d'Israël et de la Thaïlande.

${ }^{8}$ Point de presse du 19 octobre 2017 à Abidjan, sur la découverte de cas de peste porcine dans le département de Ferkessédougou et mise en place de mesures d'interdiction, de consommation et de la commercialisation de porcs de cette localité. http://www.gouv.ci/_actualite-article.php?recordID=8225. 
alternative de protéines pour les populations en milieu rural (Dindé et al., 2017).

Cependant, selon Ministère de la Santé et de l'Hygiène Publique de Côte d'Ivoire (2016), cette mobilisation de l'Etat ivoirien pour assurer une bonne nutrition, reste confrontée des contraintes, qui affectent ainsi les résultats attendus. En effet, force est de constater que près du tiers des enfants de moins de cinq ans ont un retard de croissance et les trois quarts souffrent d'anémie (Ministère de la Santé et de l'Hygiène Publique de Côte d'Ivoire, 2016). L'analyse de la situation alimentaire en Côte d'Ivoire montre que des pesanteurs socioculturelles seraient les principales causes qui empêcheraient ainsi l'adoption des bonnes pratiques nutritionnelles (Ministère de la Santé et de l'Hygiène Publique de Côte d'Ivoire, 2016). Le Plan Multisectoriel de Nutrition 2016-2020 (Ministère de la Santé et de l'Hygiène Publique de Côte d'Ivoire (2016), revèle qu'une alimentation variée et équilibrée est un préalable à une bonne santé. Mais les habitudes et pratiques alimentaires endogènes de certains groupes sociaux, sont sujettes à des normes qui interdisent la consommation d'aliments à forte valeur nutritionnelle. Par ailleurs, selon Asi and Teri (2016), ces pratiques alimentaires qui consistent à s'interdire la consommation d'un aliment, existent dans toutes les cultures et sociétés africaines et varient d'une communauté à une autre. C'est ainsi que Pagezy (2006), note que dans de nombreuses sociétés africaines, les interdits alimentaires portent particulièrement sur des espèces animales, riches en protéines de bonne qualité comme le gibier.

En Côte d'Ivoire, le poisson en plus du gibier, constitue l'une des principales sources de protéines, qui fait l'objet d'une réglementation particulière dans certaines cultures. Cette protéine (le poisson) est disponible, se conserve et se consomme sous la forme fraiche, sèche ou encore fumée. Le poisson possède des qualités organoleptiques appréciées chez les consommateurs ivoiriens et fait partie de leurs habitudes alimentaires (Akindès, 1995). Cependant, en dépit de tous ces intérêts, la consommation de certaines espèces de poissons est réglementée voire interdite dans certaines communautés ivoiriennes. Aussi, les activités de pêche sont-elles formellement proscrites par des autorités traditionnelles autochtones dans certains de leurs plans d'eau (rivières, mares ou lacs). Ces plans d'eau regorgent généralement une dense population ichtyenne constituée essentiellement de silures (Clarias gariepinus de la famille Siluridae). Dans certaines culturelles ivoiriennes, ces mesures d'interdiction d'accès aux réserves de poissons ne participent nullement à la préservation de la ressource pour les périodes difficiles. Il s'agit d'interdiction de consommation du poisson provenant de ces plans d'eau et par extension au tabou du silure pour les membres de la communauté entière. C'est notamment le cas chez les Worodougou du Centre-Nord (site de Madoba à Oussougoula par Séguéla), 
les Abron et les Koulango du Nord-Est (site de Sransi à Bondoukou) ou encore chez les Agni du Centre-Est (site de Socotè à Bongouanou) ${ }^{9}$. Cette pratique d'interdit alimentaire semble persister malgré les nombreuses situations de crises sanitaires, de besoins nutritionnels et même de développement auxquels ces communautés font face. L'engouement des différentes catégories sociales y compris les jeunes urbanisés pour le respect de cet interdit pourrait traduire une résistance de cette pratique à l'éducation moderne et aux effets de la mondialisation. Dans ce contexte, partant du cas du poisson silure du lac "Socotè 10" chez les Agni de Bongouanou, l'on est emmené à questionner les déterminants de la pratique qui interdit la consommation de ce poisson. Spécifiquement, quelles sont les connaissances et les perceptions qui soustendent cette restriction? Quelles sont les stratégies locales mises en œuvre par les autorités traditionnelles pour maintenir le respect de cet interdit alimentaire? Quelles sont les logiques sociales et sanitaires du maintien de cet interdit alimentaire à Bongouanou ?

A partir de l'exemple des Agni de Bongouanou, le présent article se propose d'analyser les déterminants sociaux, sanitaires et écologiques explicatifs de ces interdits alimentaires en s'inspirant de la théorie de l'identité sociale de Tajfel \& Turner (1986) et de la perspective matérialiste culturelle de Harris (1985). Selon la théorie de l'identité sociale, les membres du groupe tentent de se distinguer positivement en redéfinissant ou en altérant les dimensions sur lesquelles se fondent la comparaison intergroupe. La perspective matérialiste culturelle (Harris, 1985) quant à elle servira à mettre en exergue les besoins culturels auxquels répondent la mise en place de ces interdits alimentaires. Pour y parvenir, il s'agira d'analyser successivement la production de l'identité du groupe "Agni de Bongouanou" par l'usage des interdits alimentaires, ensuite, la mobilisation des questions sanitaires et de santé de reproduction comme facteurs explicatifs du maintien de ces restrictions alimentaires et enfin l'influence de cette pratique alimentaire sur l'environnement physique autour de ces communautés.

\section{Matériel et Méthodes \\ Milieu d'étude}

Cette étude a été réalisée dans le département de Bongouanou majoritairement habité par les populations du groupe ethnique Agni. Les Agni appartiennent au grand groupe ethnoculturel Akan. On les retrouve au CentreEst de la Côte d'Ivoire (Décret $\mathrm{N}^{\circ}$ 2012-612, 2012) ${ }^{11}$ dans la région du

${ }^{9}$ Cette observation est issue de l'enquête exploratoire menée dans le cadre de cette étude dans les zones, Nord, Ouest et Est de Côte d'Ivoire.

10 Socotè: Nom local attribué les autochtones Agni de Bongouanou à ce plan d'eau contenant des poissons silures interdits à la consommation

${ }^{11}$ Décret n 2012-612 du 04 juillet 2012 portant création de la Région du Moronou. 
Moronou, qui a pour capitale Bongouanou. Les enquêtes ont été menées dans quatre villages (Figure 1). Il s'agit de Kagandji, d'Agnikro (villages hôtes de la ville de Bongouanou), d'Abongoua et de Tanosso (deux villages environnants situés successivement au sud et au nord de Bongouanou). La sélection de ces villages s'est effectuée sur la base de trois critères: (i) l'organisation sociale avec la centralisation du pouvoir politique autour du Roi; (ii) la pratique de l'interdit de la consommation du poisson silure; (iii) le contexte écologique marqué par la présence du lac Socotè (Figure 2) avec une forte population de poissons silures interdits à la consommation.

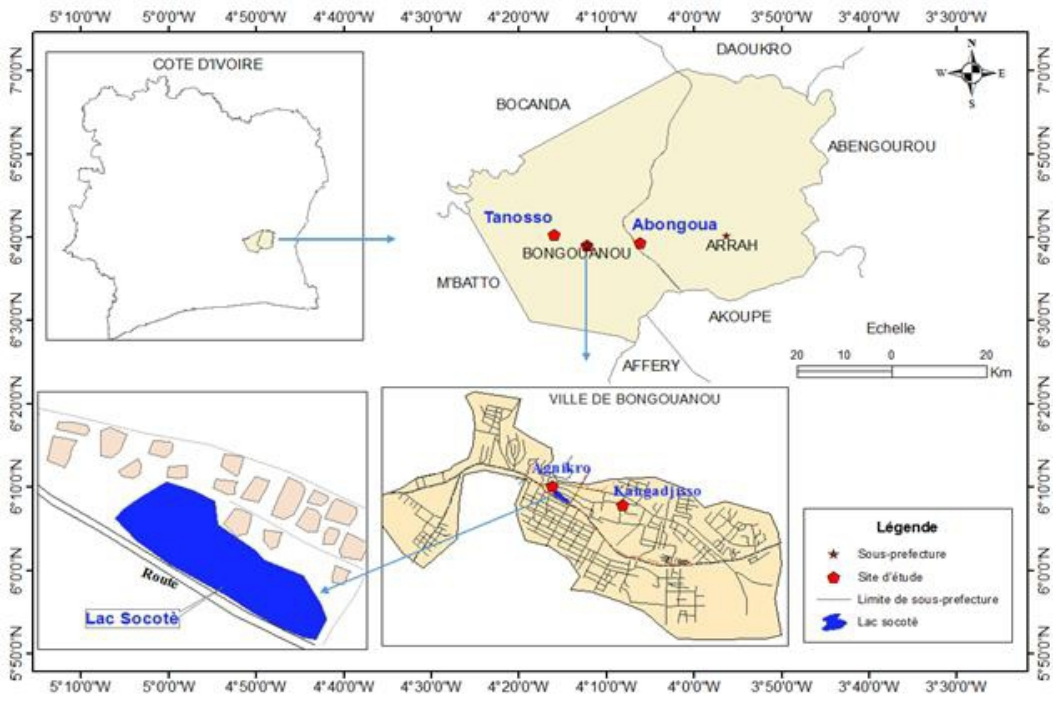

Figure 1 : Carte de la Zone d'étude

Source: BENETD

Conception: Amani Konan Romaric

Réalisation : Koné Bognan Valentin

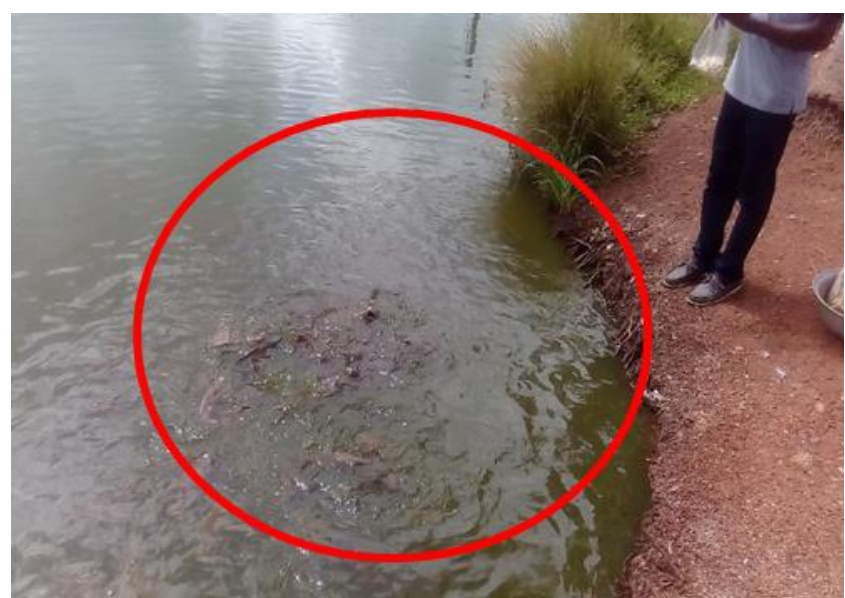

Figure 2 : Lac Socotè de Bongouanou avec affleurement des poissons silures. 


\section{Population de l'étude}

Cette étude basée sur une approche qualitative a porté sur différentes catégories d'enquêtés. Les techniques d'investigation sont l'observation, l'entretien semi-structuré et la discussion de groupe à partir d'un guide d'entretien selon Morgan (1996). L'échantillonnage a été effectué par la technique du choix raisonné pour l'identification des personnes ressources. Ainsi, les rois, les chefs de villages, les notables, des responsables de sousgroupe (jeunes et femmes) et le responsable de l'hôpital de Bongouanou ont été choisis pour les entretiens. Un total de 43 interviews individuelles et 12 discussions de groupe ont été effectuées afin de mettre en exergue les aspects relationnels et les pratiques associés aux interdits alimentaires. Le tableau1 met en exergue les caractéristiques des acteurs interviewés. Par ailleurs, le logiciel MAXQDA version 12 a servi pour l'analyse des données essentiellement qualitatives. L'analyse de contenu thématique a été mobilisée pour l'interprétation des données qui sont essentiellement sous formes de commentaires, d'opinions et de perceptions.

Tableau 1 : Récapitulatif des enquêtés

\begin{tabular}{|l|c|c|c|}
\hline \multicolumn{1}{|c|}{ Désignation } & Autochtones & Allochtones & Allogènes \\
\hline Roi et Chef de Village & 02 & 04 & 01 \\
\hline $\begin{array}{l}\text { Responsable de communauté / Porte- } \\
\text { parole }\end{array}$ & 04 & 01 & 01 \\
\hline Personne ressource du village & 01 & 04 & 04 \\
\hline $\begin{array}{l}\text { Représentante du groupe de femmes } \\
\text { du village }\end{array}$ & 04 & 04 & 04 \\
\hline $\begin{array}{l}\text { Représentant du groupe de jeunes du } \\
\text { village }\end{array}$ & 04 & Abongoua 04 \\
\hline \multicolumn{2}{|c|}{ Groupes de discussions } \\
\hline \multicolumn{2}{|c|}{ Agnikro 04 }
\end{tabular}

Les données ont par la suite été regroupées en codes pour des analyses et ont permis de positionner les unités significatives suivantes comme résultats de l'étude que nous aborderons dans l'étape suivante de notre travail. Premièrement, les interdits alimentaires participent à la sociabilité et à l'affirmation de l'identité autochtone Agni de Bongouanou. Deuxièmement, leur respect et leur maintien induisent une logique comportementale associée. Troisièmement, en plus de la construction statutaire à l'intérieur et à l'extérieur du groupe, les interdits alimentaires constituent une tentative de gestion de la question sanitaire et de conservation de la biodiversité ainsi que des services associés. 


\section{Résultats}

\section{Construction identitaire des Agni de Bongouanou autour du poisson silure du lac Socotè}

Le réfus de consommation du poisson silure du lac Socotè participe à la sociabilité et à l'affirmation de l'identité autochtone chez les Agni de Bongouanou. Ainsi, ces poissons font l'objet d'une forme d'appropriation et de spécification associée aux Agni de Bongouanou. Pour les membres de ces communautés, face à la menace de l'ordre villageois et de l'identité du groupe autochtone, la proscription du poisson silure est perçue comme une ressource de préservation identitaire et d'intégration sociale. De ce fait, le bris de cette norme alimentaire est considéré comme une source de désintégration sociale et de reniement de son identité. Dans ce sens, la plupart des enquêtés justifient les interdits alimentaires comme étant propre au territoire ou à l'espace Agni de Bongouanou. Pour ceux-ci, ces poissons sont interdits à la consommation parce qu'ils ont contribué au maintien et à la survie de leur groupe social et symbolisent ainsi leur existence et la réincarnation des leurs ancêtres. D'après un notable du village de Kangadji : " chez nous ici, il est interdit de consommer le poisson silure du lac Socotè. Tout vrai Agni de Bongouanou doit respecter cette pratique qui existe depuis la création du village. Selon nos parents, c'est grâce à ces poissons que nos ancêtres ont pu s'installer sur cet espace et ainsi de suite jusqu'à ce que nous autres soyons là aujourd'hui à vous parler » (Entretien_Notable du village_Kangadji, 26/05/17). D'après cette croyance, l'existence de la communauté est liée à la survie de ces poissons. Cette symbolique s'expliquerait par le fait que cette interdiction relève du respect de leur tradition reçue en héritage des ancêtres. Par conséquent, cet interdit assurerait la pérennité de la culture et du lien aux ancêtres. En effet, les consignes associées à l'interdit stipulent qu'il marque le fondement et la survie du groupe Agni de Bongouanou. Ainsi, le consommer s'assimilerait à un manque de reconnaissance aux ancêtres et provoquerait une certaine (vraie) culpabilité. Pour d'autres, la mort de cet animal est ressentie comme la mort d'un parent ou encore la mort du génie protecteur de la communauté. Ce poisson est de ce fait considéré comme un être spécial parce qu'intermédiaire entre les humains et Dieu ou les divinités ainsi que du monde des défunts (des ancêtres). A ce titre, le chef de village de Tanosso indique :" Chez nous les Agni de Bongouanou, ce poisson s'appelle «Edjuéblét2 c'est par lui, que nous arrivons à communiquer avec nos ancêtres. Ne pas les consommer c'est nous respecter nous mêmes et c'est aussi respecter notre culture » (Entretien_Chef de village_Tanosso, 27/05/17). Dans l'imaginaire populaire, l'interdiction de consommer édjoblé rime avec respect de la culture

\footnotetext{
12 «Edjueblé » est le nom Agni attribué au poisson silure. Ce nom est composé «Edjue » (poisson) et «blé » (noir).
} 
Agni de Bongouanou. Ce marquage culturel trace ainsi une frontière symbolique avec les autres groupes sociaux partageant l'espace culturel ivoirien. Cette façon de transmettre ce qui les lie au poisson silure du lac Socotè, relève de la préservation des constituants culturels de ce groupe. Aussi, ils précisent que ce poisson reste le trait caractéristique qui fait l'unanimité dans l'identité culturelle de la société Agni de Bongouanou.

Outre cet aspect explicatif de leur identité, deux idées sont revenues fréquemment dans le discours des répondants. La première se focalise sur le sentiment de protection ou d'assistance spirituelle de la part des ancêtres par le respect de cet interdit. Ainsi, les conséquences de son non-respect sont les multiples difficultés et malheurs. Pour eux, les exemples les plus édifiants sont entre autres, les maladies incurables, l'infertilité, les cas de déviance, les conflits inter-ethniques, le faible rendement agricole, la hausse du taux de décès chez les jeunes, etc...

La deuxième idée se focalise sur le caractère humain attribué à ce poisson. Selon les informateurs, au-delà de l'aspect animal observé, ces poissons incarnent la parenté. Par conséquent, toute personne qui consomme ce poisson, commettrait ainsi un sacrilège, car il aurait ainsi consommé un ancêtre. Un notable du village de Kangadji croit en ce lien qui les unit aux poissons du Socotè, en affirmant que : «Ces poissons sont nos ancêtres et on n'imagine pas manger ceux qui nous ont donné la vie ou encore manger nos ancêtres. Pour nous, les manger reviendrait à manger ceux qui nous protègent »(Entretien_Notable du village_Kangadji, 26/05/17). En plus de ce rapport humain évoqué par les acteurs autochtones, ils croient que ce poisson silure leur procurerait une assistance sociale particulière, notamment: 1'accès à l'emploi, les promotions professionnelles, la fécondité etc. Pour eux, cette attention spéciale s'explique par les rapports spécifiques qu'ils entretiennent avec ces poissons à la différence d'autres groupes avec qui, ils partagent le même espace social. Par ailleurs, ils ont estimé que ceux qui ne respectent pas cette norme alimentaire même en dehors du territoire de Bongouanou ne sont pas de "vrai Agni de Bongouanou”. Ceux-ci sont considérés comme des déviants et sont exposés à des sanctions (exclusion du clan ou groupe, maladie incurable, stérilité etc.,). Désirant affirmer l'importance de cette pratique d'interdit, le Roi de Bongouanou mentionne que : "Chez nous ici, le poisson silure n'est pas consommable. Aussi, tous les Agni savent qu'ils ne doivent pas manger ces poissons, car ils protègent tous les Agni de Bongouanou sans distinction » (Entretien Roi de Bongouanou, Agnikro, 25/05/17). À sa suite, un jeune autochtone d'Agnikro soutient que le poisson silure illustre plus la culture de son groupe social, que tous les autres éléments matériels: "Pour nous, le poisson silure est tellement important, qu'on ne saurait le manger pour n'importe quelle raison. Aussi, tu constateras que pour toutes situations importantes traitant de la survie des Agni de Bongouanou, on se réfère 
toujours au poisson silure du Socotè » (Focus group_Jeune du village_Agnikro, 25/05/17). La majorité des enquêtés non-autochtones a aussi affirmé que, ne pas manger le poisson silure du lac Socotè est une consigne qu'il faut respecter, même s'ils disent ne pas avoir cela comme interdit dans leur propre culture alimentaire. Dans ce sens un allochtone venu du nord de la Côte d'Ivoire affirme : "chez nous les senoufos, ce poisson n'est pas interdit alors qu'il est interdit ici chez les Agni. Nous le respectons donc pour conserver nos bons rapports avec nos tuteurs» (Entretien_Responsable de la communauté allochtone du village_Kangadji, 26/05/17). Cet extrait d'entretien met en exergue l'idée qui est régulièrement revenue chez les enquêtés et témoigne de la forte emprise de cette pratique alimentaire dans les rapports entre ceux qui les vivent. De ce fait, le respect de cet interdit alimentaire se positionne comme le canal utilisé pour conserver et entretenir les rapports avec les membres du groupe social hôte. Ainsi, pourrait-on dire que pour une meilleure cohabitation entre différents groupes sur le même espace social, ils s'attellent à réduire les différences culturelles puisque l'interaction requiert et génère une congruence de codes et de valeurs. De plus, la pratique de cet interdit permet au Agni de Bongouanou de se positionner et de légitimer leur position d'autochtone face aux autres groupes sociaux partageant le même espace social.

En le faisant, ils influencent les rapports qui les lient avec les membres des autres groupes. Ainsi, ces non-autochtones sont inscrit dans une posture de défiance voulant remmettre en cause la légitimité et les justificatifs des interdits alimentaires. Comme l'affirme un chef du village : "Une fois, les Djoulà $^{13}$, ont bravé cet interdit. Ils sont allés la nuit pêcher et consommer ce poisson. Ils sont pour la plupart tombés malades puis sanctionnés à payer des amendes auprès des autorités coutumières» (Entretien_Chef du village_Tanosso, 27/05/17). Au regard de cette affirmation, l'on note que le refus de consommer le poisson silure témoigne du travail social abattu par les responsables autochtones en vue du respect de cette pratique alimentaire. A cet effet, ces leaders autochtones produisent un sentiment d'appartenance et d'attachement communautaire, dans lequel ils se reconnaissent et auquel ils participent au quotidien. Ils ont tous indiqué que la violation de ces interdits alimentaires par l'un des leurs pourrait conduire à la perte de sa position, de son statut ou encore à son exclusion du clan. Toutefois, au regard des fonctions de construction statutaire qui se dessinent ainsi à l'intérieur et à l'extérieur du groupe Agni de Bongouanou, les interdits alimentaires sont soumis à des prescriptions comportementales chez les populations qui les vivent.

${ }^{13}$ L'appellation de Dioula (Dyola), historiquement réservée à tous les marchands soudanais, d'origine Mandé, qu'ils soient Bambara, Malinké, Dioula, Soninké, ou encore marchands ambulants est aujourd'hui attribuée à toutes les communautés du Nord de la Côte d'Ivoire, du Mali, du Burkina-Faso, de la Guinée, etc., 


\section{Du maintien de la pratique d'interdits alimentaires à une logique comportementale}

La pratique d'interdit induit des prescriptions comportementales exigées à ceux qui les vivent. Ces groupes sociaux ont un ensemble de règles qui font ressortir clairement l'importance sociale attribuée à l'interdit. Ainsi, les données recueillies montrent que certaines activités telles que la nage, la la pêche dans le lac Socotè ou encore culture de produits maraichers sur ses berges, sont formellement nterdites. Dans l'imaginaire localement partagé et transmis de génération en génération, le respect des interdits rime avec l'adoption de ces comportements spécifiques. Il est certes vrai que ces attitudes s'appliquent à tous les résidents de l'espace social Agni de Bongouanou, mais certaines varient selon le statut, le genre, l'âge ou encore selon qu'on appartienne à un sous-groupe social spécifique. En effet, à la femme, il lui est strictement interdit d'utiliser l'eau du lac pour ses activités ménagères. Les femmes enceintes doivent éviter d'être en contact avec l'eau du lac Socotè pour éviter tout contact avec le poisson. Contrairement à certaines localités, l'interdiction de consommer le poisson silure pour ces femmes s'accompagne de celle de commercialiser toutes ressources animales et végétales en provenance de ce plan d'eau.

Dès lors, la consommation ou le bris de cette restriction entraine de lourdes conséquences tant biologiques que sociales. Ainsi, du fait des croyances associées, le respect de ces normes, vise à inscrire la femme dans une posture d'évitement de tout problème lié à sa capacité de procréer. Selon une croyance localement vulgarisée, en donnant vie à un enfant, la femme gagne le respect et la considération de sa famille, mais surtout assure l'existence de sa communauté d'appartenance. Ainsi, chaque couple manifeste le désir ardent de donner naissance à autant d'enfants en évitant cet espace qui pourrait entraver l'accomplissement de ce souhait. Cela expliquerait le respect par ces femmes de toutes ces prescriptions comportementales vis-à-vis de l'interdit du lac Socotè. Une femme autochtone de Tanosso résume cette pensée en affirmant que : «Une femme doit avoir des enfants pour être respectée ici au village. Si tu es mariée et quelques années plus tard, tu n'as pas d'enfants, tu deviens la risée de ta famille et de ton village. Tu ne peux pas parler en tant que femme car tu ne connais pas les réalités de femme. Alors toutes les femmes évitent de braver l'interdit pour ne pas être des femmes stériles» (Focus group disussion_femme_Tanosso, 27/05/17). Sur la base de ces reprensentations, ces femmes établissent des liens entre la pression sociale du groupe et le respect de cet interdit alimentaire. En outre, le souci de la femme ne se limite pas seulement à la préservation de sa fécondité, de son statut de femme ou de mère, mais bien au-delà, au respect des règles et normes sociétales instituées par l'autorité coutumière.

Aussi, dans cet environnement à forte emprise de croyances mystiques et magico-religieuses, les autorités coutumières opèrent une relation implicite 
entre ces interdits et la fécondité afin d'assurer la pérennité du groupe. Dans ce sens, ces responsables établissent différentes stratégies de maintien des interdits et de sanctions en cas de non-respect de ces règles comportementales.

Dans cette logique de prévention sociale, ces leaders communautaires fixent des prescriptions comportementales qui privent ces communautés résidentes de la nourriture recommandée pour leur bon état sanitaire au profit de leur bon état social. Cette façon de faire inscrit la santé du corps social au cœur des normes alimentaires orientant les manières de faire vers ce qui est socialement attendu et accepté par le groupe social d'appartenance.

\section{Interdit alimentaire, équilibre sanitaire et santé de reproduction}

La mobilisation récurrente des questions sanitaires et de reproduction chez les Agni de Bongouanou s'est révélée comme déterminée par les interdits alimentaires. En effet, les membres des communautés autochtones enquêtés développent et établissent un lien entre la consommation des aliments interdits et l'évocation des problèmes de santé. En plus, ils justifient les cas d'infertilité par le bris d'interdit. Pour ce qui est du rapport à la santé, ils ont pour la plupart soutenu que ces restrictions contribuent à prévenir les malaises, les allergies ou même certaines maladies. Dans leur justification, ils se réfèrent régulièrement aux rapports, récits et connaissances vécus ou véhiculés dans ce sens. Ainsi, pour les informateurs, l'instauration de l'interdit est liée à l'histoire de certaines maladies ou malaises dont la récurrence a été attribuée par les premiers habitants de la localité, à la consommation de ce poisson. C'est ce que synthétise un habitant du village d'Abongoua au cours d'une discussion de groupe : "L'histoire de notre village stipule que les parents avaient constaté qu'après consommation du poisson silure du lac Socotè, ils avaient toujours des problèmes de santé tels que des boutons sur le corps, des démangeaisons ou des douleurs au ventre etc... Après avoir investigué auprès des feticheurs, il est ressorti qu'il fallait arrêter de consommer ce poisson. C'est ainsi qu'après avoir adopté cette pratique, nous avons constaté la disparition de ces maladies dans notre village» (Focus group_habitant autochtone_Abongoua, 29/05/17). Ces expériences vécues par ces derniers ont contribué à construire une image selon laquelle, ce poisson issu de ce lac était source de maladies ou de malaises. Ces connaissances associées aux interdits alimentaires sont ainsi partagées et maintenues par les autorités coutumières locales. Dans ce sens, les garants de la tradition mobilisent un ensemble de règles culturelles, qui disent-ils proviennent de cet ancêtre, être supérieur qui est le poisson silure du lac Socotè. Cet être spécial avec beaucoup plus d'autorité, les protègerait contre les malaises, les malédictions et maladies. Dans ce sens, un notable de village déclare ceci : Si tu consommes les poissons silures interdits, c'est comme si pour les ancêtres tu n'existes plus et tu ne bénéficies plus de leur protection. Pour cela tu es exposé à n'importe 
quelle sorte de problème de santé et deviens vulnérable à toutes sortes d'attaques et de maladies (naturelles ou mystiques) " (Entretien_Notable_Tanosso, 27/05/17). Ces croyances maintenues et vulgarisées s'opèrent dans des registres à la fois curatifs et préventifs. Dans une logique préventive, elles sont fondées sur cette restriction nutritionnelle conduisant à la production de normes alimentaires éloignant ainsi les résidents de l'espace social Agni de Bongouanou du poisson silure. Par ailleurs, le rapport établi entre l'interdiction de consommer le poisson silure du lac Socotè et la santé de reproduction reste un fait spontanément mentionné par la plupart des femmes. En effet, elles estiment que le respect de cette pratique alimentaire tient entre autres à la peur liée à l'infécondité et à la mauvaise santé de l'enfant. Dans l'imaginaire de celles-ci, la diminution des naissances, les difficultés rencontrées au cours des accouchements et la mauvaise santé des nouveau-nés résultent du non-respect des interdits alimentaires. Le témoignage d'une femme d'Abongoua renforce ce raisonnement : "Tu sais, il est difficile de gérer une grossesse jusqu'à l'accouchement. Alors toute femme qui souhaite avoir un enfant doit simplement éviter de consommer ce poisson. $\mathrm{Si}$ tu insistes, tu risques la stérilité ou la perte de ton enfant avant l'accouchement» (Focus group_femme_Abongoua, 27/05/17). Par cette déclaration, la santé de la femme et de l'enfant est au cœur des motivations de respect de l'interdit alimentaire. Ces croyances sont transmises au fil des générations afin de se préserver de tout risque pour la mère et l'enfant. Suivant cette logique, dans le village de Tanosso, une femme justifie ce point de vue en affirmant ceci : "Moi, de toute ma vie je n'ai jamais essayé de braver cette restriction et jusqu'à ce jour, je n'ai jamais eu de problèmes avec aucune de mes grossesses jusqu'à l'accouchement» (Entretien_femme_Tanosso, 27/05/17). En effet, cette méfiance vis-à-vis des aliments interdits est liée au fait qu'elles considèrent que la femme est plus vulnérable aux maladies mais plus spécifiquement aux "maladies dites mystiques" qui pourraient être causées par le poisson noir. Le traitement social de ces croyances opérées par ces femmes, se traduit ainsi par le refus de consommer le poisson silure afin de conserver sa bonne fécondité et d'assurer la reproduction du groupe social Agni de Bongouanou. En plus de ces questions de santé et de reproduction associées aux interdits alimentaires, on pourrait également ajouter le maintien de la conservation de la biodiversité comme un bénéfice de cette pratique alimentaire.

\section{Le maintien et la conservation de la biodiversité comme une conséquence des interdits alimentaires}

Le refus de consommer le poisson silure du lac Socotè de Bongouanou fonctionne comme un facteur de préservation de ces espèces animales de la localité. En effet, ne pas consommer le poisson silure et toutes autres espèces 
issues du lac Socotè, a favorisé une forte présence de différentes espèces du lac Socotè. Ainsi, avec l'interdiction de consommer le poisson silure, la conservation de toutes les autres espèces animales a été observée. Ce fait met en exergue le rôle joué par les peuples traditionnels à travers la pratique d'interdits alimentaires dans la protection des nombreuses espèces vivantes dans leurs écosystèmes. S'appuyant sur le cas du poisson silure de Bongouanou, il ressort que pour la survie du poisson, les autorités traditionnelles adoptent des stratégies endogènes pour préserver son existence dans le Socotè. Pour ce faire, elles procèdent à la mise en place de différentes mesures, qui interdisent la pratique de toute activité sur et autour de ce plan d'eau. C'est ainsi que tout comme le poisson silure, l'on ne pourrait savoir combien d'espèces animales ou végétales existent et vivent encore dans ce lac. Leur survie ou existence est favorisée par le groupe social autochtone Agni de Bongouanou, qui a évité de les consommer par la mise en place de cette pratique d'interdit alimentaire. En effet, s'inscrivant dans cette logique de préservation d'espaces et d'animaux interdits à la consommation, l'interdit alimentaire devient ainsi un espace d'expression écologique. L'interdiction de consommer le poisson silure du lac Socotè traduit désormais la transcendance de toute dimension alimentaire et sanitaire pour intégrer le champ écologique. Cela montre le rôle implicite de protection et de conservation de l'écosystème joué par ces communautés traditionnelles à travers la pratique d'interdit alimentaire. Dans ce sens, un jeune du village d'Agnikro affirmait ceci : «Tu sais, sans avoir vraiment fait des recherches poussées dans ce sens, mais je suis très convaincu qu'il existe des espèces animales ou végétales très rares dans ce lac...Je pourrai même recommander à des chercheurs de venir faire des recherches dans le Socotè »(Focus group_Jeune_Agnikro, 25/05/17). De cette affirmation, il ressort que la sauvegarde de la biodiversité apparait comme une conséquence inattendue de la pratique de l'interdit alimentaire.

\section{Discussion}

L'interdit alimentaire demeure une réalité vécue chez les communautés traditionnelles de Côte d'Ivoire et particulièrement chez les Agni du département de Bongouanou. Cette étude montre qu'un fait social tel que l'interdit de consommer une espèce animale ou végétale peut se poser comme un important marqueur de l'identité du groupe, mais aussi comme un canal pour la préservation de la santé et de la biodiversité.

Ainsi, dans le cadre de notre étude, il en ressort que l'interdit autour de la consommation du poisson silure s'est révélé être un trait culturel d'affirmation identitaire du groupe social Agni de Bongouanou. Il est ainsi maintenu et transmis de génération en génération et respecté par les résidents (autochtones et non-autochtones) de l'espace social Agni de Bongouanou. Bien que certains résidents non-autochtones aient des cultures alimentaires 
différentes, ils s'accordent donc à ne pas consommer le poisson silure, règle imposée par les autorités coutumières. Cette pratique d'affirmation identitaire rejoint celle décrite par Asi et Teri (2016), dans leur étude effectuée en milieu rural camerounais. Cette étude révèle que les autochtones utilisent ces interdits alimentaires pour construire et affirmer leur identité face aux mouvements d'invasions tant culturelles qu'alimentaires. Les interdits qui contribuent à se distinguer des autres, les aident à maintenir cette identité et contribuent à créer un sentiment d'appartenance. Hors de l'Afrique, cette conception est aussi présente chez les peuples de Polynésie où Mallol (2010) soutient que l'alimentation ne peut se réduire à son aspect strictement fonctionnel et nutritionnel, mais touche également aux aspects de l'identité polynésienne. L'interdiction de la consommation du chien est ainsi mobilisée par les populations pour se démarquer des autres, en affirmant ainsi leur identité dans un contexte de colonisation. De ce fait, l'interdit alimentaire est un élément central de la construction des identités. En plus de la construction et de l'affirmation identitaire, notre étude a révélé que les stratégies de maintien des interdits répondent à une volonté de positionnement et de légitimation du statut des autorités autochtones. Ce sont des règles et des comportements nonécrits répandus par les autorités coutumières de ces localités. Cette vision est soutenue par Pagezy (2006), qui dans son étude sur les N'Tomba de la République Démocratique du Congo, affirme que les interdits alimentaires répondent davantage à des considérations culturelles (arbitraire culturel) que diététiques et représentent des révélateurs du statut social. C'est dire que leur fonction sociale est importante. En outre, comparativement à l'étude de Asi and Teri (2016) au Cameroun, la monopolisation d'animaux ou parties d'animaux par une catégorie de personnes ne se dégage pas comme une caractéristique importante de restriction alimentaire. C'est plutôt, la fonction sociale dédiée à cet interdit qui joue un rôle important dans la construction de ces interdits alimentaires. A l'instar des sociétés africaines, les interdits alimentaires concernent dans beaucoup de cas, les espèces animales que l'on retrouve dans l'environnement immédiat. Celui des Agni de Bongouanou se focalise sur le poisson silure du Socotè, lac qui traverse les deux villages Agnikro et Kangadjisso. Ce point de vue se rapproche de celui de Pagezy (2006), qui estime que les interdits alimentaires concernent plus particulièrement des espèces animales, riches en protéines de bonne qualité, comme le gibier. A ce niveau, ce résultat révèle qu'en plus de mobiliser cette pratique alimentaire pour affirmer leur appartenance à ce groupe social, le refus de consommer le poisson silure fonctionne comme une démonstration de l'unicité de ce groupe face aux non-autochtones.

Il est ressorti de cette étude que l'interdiction de consommer le poisson silure contribue à la gestion de la santé de ceux qui les observent. Dans l'imaginaire collectif des Agni de Bongouanou, le respect de l'interdit du 
poisson silure permet de maintenir la bonne santé et surtout conserver la fertilité au sein du couple, ce qui va concourir à assurer la survie du groupe social. Ainsi, est-il revenu que le respect de la restriction de consommer le poisson silure sert à prévenir des malaises, des maladies ou encore pour maintenir la fertilité et faciliter les accouchements. C'est dans ce sens que Meyer-Rochow (2009), affirme qu'il existe des tabous alimentaires qui ont une base scientifique, tels que ceux associés aux réactions allergiques. Dans ce cas, l'imposition de l'interdit ferait suite aux expériences morbides ou aux cas d'allergies constatés après la consommation d'une espèce animale ou végétale. L'aspect socio-sanitaire de l'interdit concerne le plus souvent les femmes et les enfants. Une étude sur les guérisseurs traditionnels du Nigéria fait dire à Odebiyi (1989), que les maladies de l'enfance sont parfois liées au non-respect des interdits alimentaires. Au Sénégal, Guèye and Bessei (1995) ont également montré que pour éviter certaines maladies infantiles, les femmes étaient restreintes à la consommation des produits avicoles. Cette étude apporte un éclairage sur les déterminants de fertilité et de santé de reproduction, siège du jeu des croyances, qui justifient le maintien et le respect strict des interdits alimentaires chez les femmes. Pour elles, les malaises, les fausses couches ou encore la perte précoce des enfants sont liés à la désobéissance à cette restriction alimentaire comme l'affirme Yoro (2012) dans son étude relative aux Bété de Côte d'Ivoire.

Cependant, contrairement à l'étude de Pagezy (2006), la variable sanitaire ne se dégage pas comme étant un déterminant de construction ou de maintien des interdits alimentaires chez les Agni de Bongouanou. C'est plutôt leur fonction symbolique et sociale, qui motive l'application et la mise en place de ces interdits alimentaires, qui est applicable spécifiquement aux couches dites vulnérables. Mengesha and Ayele (2015) reconnaissent d'ailleurs, que les tabous alimentaires sont scientifiquement infondés et basés sur des croyances attribuées à une culture spécifique et discriminent certains membres de la société tels que les femmes et les jeunes. Pagezy and Garine (1990) soulignent que même si on associe des maladies ou malaises au nonrespect ou à la violation des interdits alimentaires, les femmes enceintes et allaitantes auxquelles ces restrictions s'imposent le plus souvent sont biologiquement vulnérables et ont des besoins accrus en protéines pour la grossesse, l'allaitement et la croissance.

Enfin de cette étude, il ressort que l'interdit alimentaire peut avoir une fonction de préservation de la biodiversité. Le poisson silure apparait comme un puissant agent de contrôle de la société, mais aussi de la gestion des ressources naturelles chez les Agni de Bongouanou. Par l'observation de cette restriction alimentaire, l'espace physique hébergeant ce poisson silure est ainsi préservé avec toutes les espèces qui peuvent s'y retrouver. C'est ce que Mcdonald (1977) ; Colding \& Folke (1997) et Begossi et al. (2004) abordent 
dans leurs études en relevant que les interdits alimentaires ont un arrière-plan écologique. En effet, tout comme la protection du poisson silure par la conservation du lac Socotè, les cas de l'interdiction de consommation du singe dans d'autres localités montrent leur contribution dans la protection de la forêt entière avec toutes les espèces que l'on pourrait y retrouver. Ainsi, l'interdit alimentaire devient-elle une pratique écologique, et peut être attribuée à la culture alimentaire des communautés Yacouba de Man à l'Ouest de la Côte d'Ivoire, avec la conservation du singe, que l'on apercevoit dans la forêt et les collines de Gbêpleu. La localité de Soko à l'Est de la Côte d'Ivoire présente un cas similaire avec ses singes sacrés retrouvés dans l'espace immédiat des communautés résidentes.

Si dans certaines communautés, les interdits alimentaires peuvent être une conséquence symboliques ou mythologiques, force est de reconnaître les motifs utilitaristes attribués à ceux-ci. Ainsi, les restrictions ou pratiques d'interdits alimentaires sont-elles certes subtiles et moins clairement définies comme stratégies de conservation des ressources naturelles, mais s'avèrent très efficace dans la gestion de la biodiversité des espaces peuplés par les communautés. Par ailleurs, dans un contexte où l'environnement physique immédiat demeure le lieu privilégié de production des ressources alimentaires, la limitation d'utilisation de certaines de ces ressources reste un moyen incontournable de protection de la biodiversité locale. Ainsi, ressort-il clairement de cette étude, que la conservation de certaines espèces végétales ou animales à travers la pratique d'interdits alimentaires pourrait être valorisée en vue de participer culturellement à la conservation de la biodiversité et contribuer par ailleurs au bien-être des communautés .

Cependant, conformément à la conclusion de Harris (1985), l'interdit alimentaire serait, selon nos résultats, justifiable d'une analyse en termes d'avantages ou à la satisfaction d'un besoin, mais pas seulement pour les mêmes raisons qu'il évoque. En effet, il évoque les besoins d'ordre matériel culturel et écologique des interdits. Toutefois, si sur l'aspect écologique ou encore matériel culturel l'on se rejoint, l'une des raisons évoquées par les Agni de Bongouanou reste l'utilisation de cet interdit alimentaire pour la prévention des risques sanitaires et pour la santé de reproduction. Ainsi, l'ordre social vat-il de pair avec le respect des interdits alimentaires et contribue à assurer le bien-être social de la communauté Agni de Bongouanou.

\section{Conclusion}

$\mathrm{Au}$ terme de ce travail traitant des déterminants de la pratique des interdits alimentaires chez les Agni de Bongouanou, il ressort qu'au-delà du symbolisme associé, cette pratique répond à des besoins communautaires. Ainsi, sa pratique se présente comme une affirmation identitaire, d'obéissance et de soumission aux règles et normes qui régissent cette communauté. Outre 
ces aspects, le maintien de ces interdits dans ce groupe social, reste du ressort des autorités coutumières, qui les imposent à travers la mise en oeuvre de différentes stratégies et règles comportementales. En effet, le respect de cette logique comportementale par les résidents, permet d'une part, d'affirmer l'autorité de ceux qui les imposent, et d'autre part, de conserver le contact avec la culture hôte Agni de Bongouanou. Par ailleurs, la pratique des interdits alimentaires répond à des besoins de protection contre le mal, les mauvais sorts, de non-procréation, de lutte contre les maladies et les malaises. Aussi, il ressort qu'étudier les interdits alimentaires dans l'espace rural de la Côte d'Ivoire, particulièrement chez les populations de Bongouanou, est un canal de révélation des valeurs et des normes sociales associées à la pratique alimentaire d'une part. D'autre part, cette pratique se révèle être un moyen endogène de pratique écologique pour la préservation des ressources naturelles. Toutefois, il est important de souligner que, l'interdit alimentaire pourait se justifier en termes d'avantages ou à la satisfaction d'un besoin. Cependant, s'il est vrai que les besoins d'ordre matériel culturel et écologique ont déjà été avancés dans certaines études, les principales justifications chez les Agni de Bongouanou restent la prévention des risques sanitaires, l'affirmation identitaire, la santé de reproduction et la conservation de la biodiversité. Dans ce sens, la réflexion sur la question de la nutrition en Côte d'Ivoire doit prendre en compte le contexte environnemental, lieu privilégié de production des ressources alimentaires afin d'intégrer les bouleversements climatiques mais surtout la contribution des pratiques alimentaires dans la conservation de la biodiversité locale.

\section{Remerciements}

Cette étude a été réalisée avec la subvention du Programme d'Appui Stratégique à la Recherche Scientifique (PASRES), Projet $N^{\circ} 100$. Cet article s'inscrit dans le cadre de l'initiative DELTAS Africa Initiative [Afrique OneASPIRE / DEL-15-008]. Afrique One-ASPIRE est financé par un consortium de bailleurs de fonds, dont l'Académie africaine des sciences, l'Alliance pour l'accélération de l'excellence scientifique en Afrique, l'Agence du Nouveau partenariat pour la planification et la coordination du développement en Afrique (NEPAD), le Wellcome Trust [107753 / A / 15 / Z] et la coopération Britannique. Par ailleurs, nous tenons à remercier tous ceux qui ont participé a cette étude et accepté de répondre à nos questions. Nous manifestons notre reconnaissance à tous ces responsables de structures, d'associations et de communautés qui ont facilité la réalisation de cette étude dans leur localité.

\section{References:}

1. Akindès F (1995). Dévaluation et alimentation à Abidjan (Côte d'Ivoire). Cahiers de la Recherche Développement: pp 36-39. 
2. Asi LN \& Teri DT (2016). Influence of food taboos on nutritional patterns in rural communities in Cameroon. International Review of Social Research 6: pp 35-39.

3. Begossi A, Hanazaki N \& Ramos RM (2004). Food chain and the reasons for fish food taboos among Amazonian and Atlantic Forest fishers (Brazil). Ecological Applications 14: pp 1334-1343.

4. Colding J \& Folke C (1997). The relations among threatened species, their protection, and taboos. Conservation ecology,: pp 1-19.

5. Décret $N^{\circ} 2012-612$ (2012). Décret $N^{\circ} 2012-612$ du 04 juillet 2012 portant création de la Région du Moronou. Journal officiel de la République de Côte d'Ivoire: 2 p.

6. Dindé AO, Mobio AJ, Konan AG, Fokou G, Yao K, Esso ELJC, Fantodji A, Koussemon M \& Bonfoh B (2017). Response to the Ebolarelated bushmeat consumption ban in rural Côte d'Ivoire. Agriculture \& Food Security 28: pp 1-9.

7. FAO I (2016) WFP (2015). The State of Food Insecurity in the World 2015. Meeting the 2015 international hunger targets: taking stock of uneven progress. Food and Agriculture Organization Publications, Rome, Consulté le 23/09/2017 sur le site http://www.fao.org/contactus/licence-request/en/.

8. Guèye E-HF \& Bessei W (1995). About food bans and taboos on poultry products in Senegal. Der Tropenlandwirt-Journal of Agriculture in the Tropics and Subtropics 96: pp 97-109.

9. Harris M (1985). Good to Eat: Riddles of Food and Culture, Simon and Schuster,, New York, 289p.

10. Mallol CS (2010). Manger du chien à Tahiti: une affirmation identitaire? Anthropozoologica 45: pp 157-172.

11. Mcdonald DR (1977). Food taboos: a primitive environmental protection agency (South America). Anthropos 72: pp 734-748.

12. Mengesha AD \& Ayele TT (2015). The Impact of Culture on the Nutritional Status of Children and Mothers Durrinng Recurring Food Insecurity: The Case of Boreicha Woreda (SNNPRS). American Journal of Educational Research 3: pp 849-867.

13. Meyer-Rochow VB (2009). Food taboos: their origins and purposes. Journal of Ethnobiology and Ethnomedicine 5: pp 1-10.

14. Ministère de la Santé et de l'Hygiène Publique de Côte d'Ivoire (2016). Plan National Multisectoriel de Nutrition. 34p.

15. Morgan DL (1996). Focus groups as qualitative research Sage publications 16: PP 32-46.

16. Odebiyi AI (1989). Food taboos in maternal and child health: the views of traditional healers in Ile-Ife, Nigeria. Social Science \& Medicine 28: pp 985-996. 
17. Pagezy H (2006). Alimentation et croissance: Faut-il condamner les interdits alimentaires? Antropo 11: pp 119-127.

18. Pagezy H \& Garine ID (1990). Food and nutrition among "high-risk" groups. in Food and nutrition in the African rain forest. S. Bahuchet and I. De Garine edn. Unesco/MAB, C.M. Hladik, S. Bahuchet and I. De Garine (Eds), 73-77., Paris.

19. Tajfel H \& Turner J (1986). The social identity theory of intergroup behavior,: Psychology of intergroup relations (ed. by SeA In Worchel, W.G (Eds.),) Nelson-Hall, Chicago, pp. pp 7-24.

20. Yacouba K (2007). Contribution à l'évaluation de l'incidence socioéconomique de la grippe aviaire en Cöte d'Ivoire au cours de l'année 2006., Vol. Veterynary Phd: Faculté de Médecine, de Pharmacie et d'Odonto Stomatologie (ed. Université Cheik Anta Diop. Pages 151, Dakar, p. 151 p.

21. Yoro BM (2012). Maladies honteuses et recours aux soins chez les Bété (Côte d'Ivoire). European Journal of Scientific Research 89: pp 225-236. 